\title{
PROBLEMAS METODOLÓGICOS NA OBTENÇÃO DA CURVA DE RETENÇÃo DA ÁGUA PELO SOLO ${ }^{1}$
}

\author{
S.O. MORAES ${ }^{2}$; P.L. LIBARDI ${ }^{2}$ \\ Departamento de Fisica e Meteorologia, ESALQ/USP, C.P.9, CEP: 13418-900 - Piracicaba,SP. \\ D. DOURADO NETO \\ Departamento de Agricultura, ESALQ/USP, C.P.9, CEP: 13418-900 - Piracicaba,SP.
}

RESUMO: Para estudar a variabilidade tanto metodologica quanto espacial da Curva de Retenção de Água no Solo, coletaram-se amostras de solo com estrutura indeformada à profundidade de $25 \mathrm{~cm}$ em uma área de Terra Roxa Estruturada Latossolica (Rhodic Kanhapludalf) em Piracicaba,SP. A amostragem obedeceu a um arranjo com espaçamento regular de cinco metros, resultando numa malha quadriculada de vinte e cinco linhas e dez colunas, totalizando duzentos e cinquienta pontos. Elaboraram-se 250 Curvas de Retenção por secamento, utilizando-se as tensões de $5 \times 10^{2} ; 1 \times 10^{3} ; 6 \times 10^{3} ; 1 \times 10^{4}$ Pa (Funil de Placa Porosa) e $3 \times 10^{4} ; 8 \times 10^{4} ; 3 \times 10^{5}$ e $1,5 \times 10^{6}$ Pa (Câmara de Pressão de Richards), totalizando oito pontos por curva. Analisou-se a variabilidade metodológica para os vários valores de tensão considerados, principalmente à luz da termodinâmica pertinente. Os resultados evidenciaram que os problemas analíticos são de tal ordem que podem levar a interpretaçōes errôneas, comprometendo a qualidade de experimentos ou mesmo a comparabilidade de resultados provenientes de laboratórios diversos. Exemplificando, $43 \%$ das amostras apresentaram resultados inconsistentes, com valores de umidade à base de volume à $3 \times 10^{5} \mathrm{~Pa}$ menores que a $1,5 \times 10^{6} \mathrm{~Pa}$, o que na prática poderia levar, dentre outros, a problemas de subestimativa nos valores de água disponível.

Descritores: curva de retenção da água pelo solo, variabilidade, termodinâmica da água no solo.

\section{METHODOLOGICAL PROBLEMS OF THE SOI-WATER RETENTION CURVE}

ABSTRACT: Undisturbed soil samples of a "Terra Roxa Estruturada Latoss6lica" (Rhodic Kanhapludalf) were taken in Piracicaba,SP from the $25 \mathrm{~cm}$ depth, in order to study both methodological and spatial variabilities of the soil water retention curve. The sampling followed a $5 \mathrm{~m} \times 5 \mathrm{~m}$ grid of 10 columns and 25 lines, resulting 250 collecting points. With these samples, 250 retention curves were obtained using porous plate funnels (tensions of $5 \times 10^{2} ; 1 \times$ $10^{3} ; 6 \times 10^{3}$ and $1 \times 10^{4} \mathrm{~Pa}$ ) and Richards pressure chambers (tensions of $3 \times 10^{4} ; 8 \times 10^{4} ; 3 \times 10^{5}$, and $1.5 \times 10^{6} \mathrm{~Pa}$ ). The methodological variability was analyzed mainly in the light of the pertinent thermodynamics, using several values of tension. Results have shown that the analytical problems are such that they can lead to erroneous interpretrations, affecting the quality of the experiment and also the comparison of results from several laboratories. For instance, $43 \%$ of the samples showed non consistant results, with values of volumetric soil-water content smaller at the tension of $3 \times 10^{4} \mathrm{~Pa}$ than at the tension of $1.5 \times 10^{6} \mathrm{~Pa}$, which, from the practical point of view, could, lead to subestimated values of available water.

Key words: Soil-water retention curve, variability, thermodynamics of soil-water.

\section{INTRODUÇÃO}

A variabilidade espacial das propriedades físico-hídricas do solo é um dos fatores que pode tornar variável o manejo do solo e da água numa agricultura irrigada. Embora o reconhecimento da variabilidade possa remontar ao amanhecer da
Ciência do Solo, o enfrentamento dessa variabilidade não apenas como mais um elemento típico da paisagem parece ser privilégio do presente século.

Neste sentido, SILVA (1988) relaciona alguns trabalhos, particularmente da primeira metade do século, que expressavam a preocupação

\footnotetext{
' Parte da Tese de Doutorado do primeiro Autor, junto ao Curs de Pós-Graduação em Solos e Nutrição de Plantas da ESALQ/USP.

${ }^{2}$ Bolsista do CNPq.
} 
de se levar em conta a variabilidade espacial em experimentos de campo.

Dentre as propriedades físico-hídricas, uma de difícil caracterização tanto pelo tempo que se consome nas análises quanto pela intrínseca modificação da amostra devido à histerese, é a Curva de Retenção da Água no Solo, abreviadamente, curva de retenção, que expressa a relação entre a umidade do solo, a base de massa ou volume, e o potencial mátrico correspondente (CHILDS, 1940), a qual é extremamente importante em quaisquer estudos que envolvem água no solo.

A retenção da água no solo é a fetada por uma série de fatores, que vão desde a distribuição relativa do tamanho, da forma e do arranjo das partículas do solo (SALTER \& WILLIANS, 1965; REEVE et al., 1973; SHARMA \& UEHARA, 1968) até a composição e concentração de solutos na solução do solo, no caso deste conter argilas expansivas (EL-SWAIFY \& HENDERSON, 1967; THOMAS \& MOODIE, 1962). Embora estes fatores possam ser altamente variáveis em uma mesma unidade pedológica, quando se analisa uma amostra das várias repetições consideradas, esta também pode sofrer alterações na curva de retenção, seja por fenômenos de histerese, às vezes tão significativa quanto a própria variabilidade espacial do solo, ROYER \& VACHAUD (1975), seja por diferenças de temperatura no ato da elaboração da curva de retenção, isto é, com a amostra no funil ou na câmara de pressão.

A influência da temperatura deve-se ao fato de que a intensidade das forças que retem a água no solo a um dado potencial é dependente da temperatura. A tensão superficial diminui com a temperatura, o que leva à uma redução no teor de água a um dado potencial. Os efeitos da temperatura sobre as forças de adsorção são um pouco obscuros (KLUTE, 1986) mas novamente parece que um aumento na temperatura leva a uma redução no teor de água a um dado potencial.

$\mathrm{Na}$ década de 60 , principalmente, uma série de pesquisadores se dedicou a estudar os efeitos da temperatura sobre a tensão superficial, mostrando que apenas esses efeitos não são suficientes para explicar as diferenças observadas (PECK, 1960; WILKINSON \& KLUTE, 1962; CHAHAL, 1963; CHAHAL, 1965 e CHAHAL, 1966), mas que também as alterações devido à temperatura, no volume de ar aprisionado na amostra, têm que ser levadas em conta (CHAHAL, 1963), além da dependência do próprio volume de água com a temperatura (PECK, 1960).
Em vista do exposto, o presente trabalho objetiva proporcionar maiores conhecimentos sobre curva de retenção em situações de flutuação de temperatura, bem como indicar os cuidados pertinentes na sua elaboração.

\section{MATERIAL E MÉTODOS}

A área estudada está localizada geograficamente a $22^{\circ} 43^{\prime}$ de latitude sul e $475^{\circ} 5^{\prime}$ de longitude oeste, com altitude de $580 \mathrm{~m}$ acima do nível do mar, situada no campo experimental do Departamento de Física e Meteorologia, campus da Escola Superior de Agricultura "Luiz de Queiroz", Universidade de São Paulo, município de Piracicaba, Estado de São Paulo, Brasil.

O solo foi classificado como Terra Roxa Estruturada Latossólica (VIDAL TORRADO', 1991) correspondente ao Rhodic Kanhapludalf (Soil Survey Staff, 1990) da classificação americana.

O delineamento utilizado proporcionou uma amostragem sistemática (WEBSTER, 1977; PETERSEN \& CALVIN, 1986) de volumes indeformados de solo, obedecendo a um arranjo com espaçamento regular de 5 metros, resultando numa malha quadriculada de 25 linhas e 10 colunas, totalizando 250 pontos.

Foram coletadas 250 amostras de solo com estrutura indeformada, à profundidade de 25 $\mathrm{cm}$, as quais foram utilizadas para obtenção das respectivas umidades, às tensões de $5 \times 10^{2} ; 1 \times$ $10^{3} ; 6 \times 10^{3} ; 1 \times 10^{4} ; 3 \times 10^{4} ; 8 \times 10^{4} ; 3 \times 10^{5} \mathrm{e}$ $1,5 \times 10^{6} \mathrm{~Pa}$ e da densidade do solo e posterior análise estatística.

Para extração e confinamento da amostra utilizaram-se anéis de alumínio com uma das bordas cortantes (BLAKE \& HARTGE 1986), com cerca de $3,0 \mathrm{~cm}$ de altura e $4,7 \mathrm{~cm}$ de diâmetro, dimensões estas que estão dentro dos padrões geralmente utilizados (KLUTE, 1986).

Dependendo da tensão necessária, os equipamentos utilizados foram funil de placa porosa (HAINES, 1930) e câmara de pressão de Richards (RICHARDS \& FIREMAN, 1943).

Assim, quatro pontos foram determinados por sucção $\left(5 \times 10^{2} ; 1 \times 10^{3} ; 6 \times 10^{3} \mathrm{e} 1 \times 10^{4}\right)$; e quatro por pressão $\left(3 \times 10^{4} ; 8 \times 10^{4} ; 3 \times 10^{5}\right.$ e 1,5 $x 10^{6}$ ).

A câmara de pressão de Richards utilizada $\varepsilon$ o equipamento padrão fabricado pela Soil Moisture Equipment Corp. O funil de placa

*VIDAL TORRADO, P. Comunicação pessoal, 1991. 
porosa foi confeccionado com capacidade para dezessete amostras nas dimensões mencionadas, em contra partida ao funil convencional fabricado pela JENA GLASS (FREIRE, 1975; MOTA, 1976) com capacidade para uma amostra.

No presente trabalho determinaram-se curvas de retenção por secagem, mais propriamente falando, para cada amostra determinou-se a curva principal de drenagem uma vez que não se iniciou a partir de uma saturação completa de amostra, mas de um valor $\theta_{0}<\theta_{\mathrm{a}}$, (KLUTE 1986, BALL \& HUNTER, 1988).

O fluido utilizado para molhamento foi água destilada e deaerada, através de agitação intermitente sob vácuo. $O$ tempo de molhamento pelo processo de embebição foi de 48 horas por lote de amostras distribuídas de forma a levar-se 24 horas para a elevação do nível da lâmina de água até a borda superior da amostra e o tempo restante a fim de que mais bolhas de ar pudessem ser libertadas (KLUTE, 1986; BALL \& HUNTER, 1988).

Nos funis, as amostras foram embebidas diretamente. No inicio, com uma lâmina de cerca de $0,2 \mathrm{~cm}$, as amostras eram posicionadas nos funis, elevando a lâmina através da adição de água pelo tubo de vidro, isto é, a água atravessava inicialmente a placa e desta ia para as amostras. Nas câmaras de pressão as amostras foram embebidas em bandejas plásticas, durante o mesmo tempo e depois levadas à câmara com as respectivas placas porosas.

Tanto para os funis, quanto para as câmaras de pressão, o momento de equilibrio foi determinado pelo cessamento da drenagem, verificado visualmente pela ausência de gotas de água e formação de menisco nos tubos de saída (KLUTE, 1986).

Após o equilibrio, as amostras eram retiradas do equipamento, determinava-se as massas por pesagem e, quando não havia necessidade de se refazer as colagens de lenço de papel, fato que ocorreu durante todo o período de análises, devido ao desgaste do material, as amostras sofriam novo molhamento $e$ o processo tinha continuidade.

Ao final das determinações, as amostras foram secas em estufa a $105-110^{\circ} \mathrm{C}$, por 48 horas e após o equilibrio térmico em dessecador, sendo determinadas as massas de sólidos e a tara (compreendendo cilindro de alumínio, cola e lenço de papel). Em seguida, procedia-se aos cálculos de densidade do solo, umidades a base de massa e de volume. Com apenas duas modificações (fluido e tempo de molhamento), este é basicamente o processo de obtenção da curva de retenção contido em EMBRAPA (1979).

\section{RESULTADOS E DISCUSSÃO}

A Figura 1 mostra a comparação entre o número de amostras onde ocorreram valores de umidade à base de volume correspondente à tensão de $5 \times 10^{2} \mathrm{~Pa}$ maiores (Figura 1, a), menores (Figura 1, b) e iguais (Figura 1, c) aos valores de umidade correspondente a $1 \times 10^{3} \mathrm{~Pa}$.

Observa-se inicialmente que para a maioria dos casos há uma igualdade entre os valores de umidade a $5 \times 10^{2} \mathrm{e} 1 \times 10^{3} \mathrm{~Pa}$, fato este realçado na TABELA 1 que apresenta alguns parâmetros estatísticos das variáveis estudadas, entre eles a média e desvio para os dois valores de tensão mencionados. Verifica-se então pela TABELA 1, que os valores da média para estas tensões, bem como os valores respectivos do desvio, não permitem a distinção entre as duas umidades.

KLUTE (1986) explica esse patamar para baixos valores de tensão pela rigidez estrutural natural da amostra.

No entanto, uma segunda hipótese seria - próprio ato de aparar a amostra. Tal procedimento que é feito quando a amostra se encontra com uma umìdade próxima à "capacidade de campo" (MORAES, 1991) poderia produzir um "espelhamento" da superfície do solo, isto é, não apenas uma interrupção brusca na continuidade de poros nas faces expostas, inevitável pela própria necessidade de análise, mas também um deslizamento de partículas sobre as superfícies, facilitado pela umidade elevada, provocando o aspecto espelhado.

Esse deslizamento poderia levar a um aumento de rigidez estrutural na superfície da amostra, dificultando a entrada de ar o que consequentemente, exige uma pressão de ar limiar, ou seja um valor de entrada de ar, à partir do qual a água começasse a ser retirada entre $5 \times 10^{2} \mathrm{e} 1 \mathrm{x}$ $10^{3} \mathrm{~Pa}$. Uma terceira hipótese para o fenômeno diz respeito ao diâmetro dos poros que seriam esvaziados quando da aplicação das diversas pressões.

A partir da fórmula da ascensão capilar, $h=2 \sigma / r \rho g$, quie se torna $d \cong 3 / h^{\circ}$ quando se considera água a $25^{\circ} \mathrm{C}$, onde $\mathrm{d} \varepsilon$ o diâmetro do poro em milímetros e h é a tensão expressa em centímetros 
TABELA 1 - Parâmetros estatísticos das variáveis umidade a base de massa (U.g/g), densidade do solo (Ds, $\left.\mathrm{g} / \mathrm{cm}^{3}\right)$, e umidade a base de volume $\left(\theta, \mathrm{cm}^{3} / \mathrm{cm}^{3}\right)$. Tamanho da amostra: 250 .

\begin{tabular}{|c|c|c|c|c|c|c|}
\hline \multirow[b]{2}{*}{ Variável } & \multirow{2}{*}{$\begin{array}{c}\text { Potencial } \\
\text { Mátrico } \\
(\mathrm{Pa})\end{array}$} & \multicolumn{2}{|c|}{ Valores } & \multirow[b]{2}{*}{ Média } & \multirow{2}{*}{$\begin{array}{l}\text { Desvio } \\
\text { Padrão }\end{array}$} & \multirow{2}{*}{$\begin{array}{l}\text { Coeficiente } \\
\text { Variação \% }\end{array}$} \\
\hline & & Mínimo & Máximo & & & \\
\hline \multirow[t]{8}{*}{$\mathbf{U}$} & $5 \times 10^{2}$ & 0,214 & 0,538 & 0,315 & 0,031 & 10 \\
\hline & $1 \times 10^{3}$ & 0,229 & 0,540 & 0,314 & 0,031 & 10 \\
\hline & $6 \times 10^{3}$ & 0,217 & 0,326 & 0,278 & 0,021 & 7 \\
\hline & $1 \times 10^{4}$ & 0,213 & 0,315 & 0,267 & 0,020 & 7 \\
\hline & $3 \times 10^{4}$ & 0,165 & 0,301 & 0,252 & 0,019 & 8 \\
\hline & $8 \times 10^{4}$ & 0,185 & 0,308 & 0,235 & 0,019 & 8 \\
\hline & $3 \times 10^{5}$ & 0,157 & 0,272 & 0,208 & 0,021 & 10 \\
\hline & $1,5 \times 10^{6}$ & 0,154 & 0,240 & 0,199 & 0,020 & 10 \\
\hline Ds & - & 1,272 & 1,727 & 1,490 & 0,071 & 5 \\
\hline \multirow[t]{8}{*}{$\theta$} & $5 \times 10^{2}$ & 0,316 & 0,685 & 0,468 & 0,031 & 7 \\
\hline & $1 \times 10^{3}$ & 0,338 & 0,686 & 0,466 & 0,031 & 7 \\
\hline & $6 \times 10^{3}$ & 0,335 & 0,464 & 0,413 & 0,022 & 5 \\
\hline & $1 \times 10^{4}$ & 0,327 & 0,450 & 0,397 & 0,021 & 5 \\
\hline & $3 \times 10^{4}$ & 0,238 & 0,435 & 0,375 & 0,023 & 6 \\
\hline & $8 \times 10^{4}$ & 0,283 & 0,461 & 0,349 & 0,025 & 7 \\
\hline & $3 \times 10^{5}$ & 0,241 & 0,402 & 0,310 & 0,028 & 9 \\
\hline & $1,5 \times 10^{6}$ & 0,232 & 0,361 & 0,296 & 0,027 & 9 \\
\hline
\end{tabular}

de coluna de água com a qual a água do solo entra em equilíbrio, $\epsilon$ possível calcular o diâmetro dos poros esvaziados a uma dada tensão. Medindo-se ainda o volume de água removida a uma dada tensão, tem-se o volume de poros do tamanho indicado por esta tensão (LEAMER \& LUTZ, 1940; DANIELSON \& SUTHERLAND, 1986).

Assumindo-se que a equação da ascensão capilar é aplicável ao presente sistema solo-água, pode-se calcular os valores aproximados de suç̧ão, para os quais a água é retirada dos poros. A partir então dos valores médios das umidades a base de volume da TABELA 1, obter-se-ia o conjunto de valores mostrado acima e representados de forma esquemática na Figura 2.

Como praticamente não houve esvaziamento de poros entre as tensões $5 \times 10^{2}$ e 1 $\times 10^{3}$, supondo que as duas hipóteses anteriores não sejam válidas, seria de se esperar que o diâmetro máximo dos poros seria da ordem de $0,3 \mathrm{~mm}$, estando na verdade as maiores proporções de vazios contidas entre os diâmetros $0,3 \mathrm{~mm}(1 \mathrm{x}$ $\left.10^{2} \mathrm{~Pa}\right)$ a $0,05 \mathrm{~mm}\left(6 \times 10^{3} \mathrm{~Pa}\right)$ e $0,0037 \mathrm{~mm}(8 \times$ $\left.10^{4} \mathrm{~Pa}\right)$ a $0,0010 \mathrm{~mm}\left(3 \times 10^{5} \mathrm{~Pa}\right)$. 


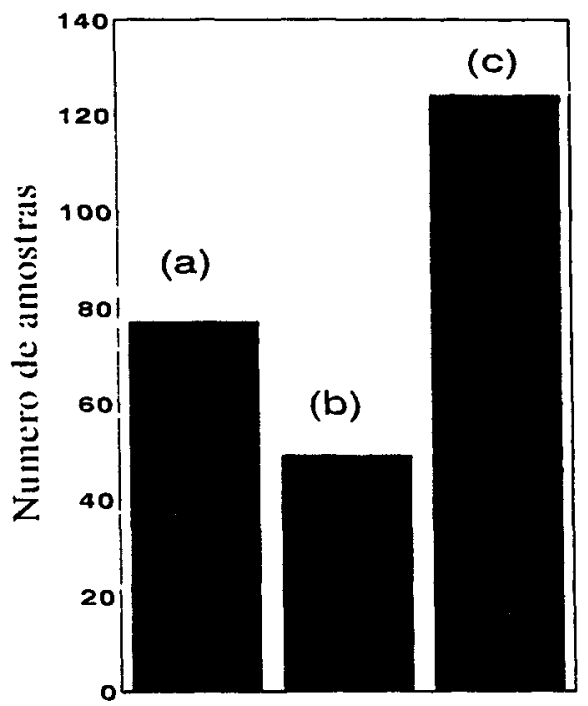

Figura 1. Comparação entre o número de amostras onde ocorreram valores de umidade correspondente a $5 \times 10^{2} \mathrm{~Pa}$ maiores (a), menores (b) e iguais (c) aos valores de umidade correspondente a $1 \times 10^{3} \mathrm{~Pa}$.

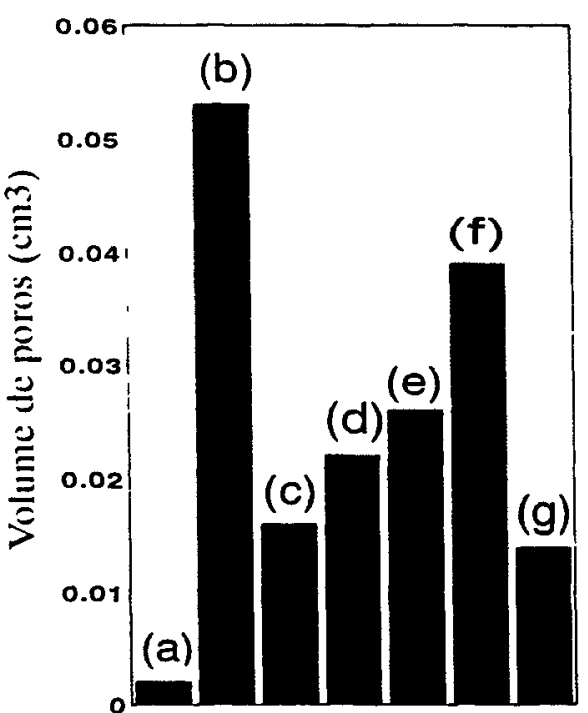

Figura 2 - Volume de poros $\left(\mathrm{cm}^{3}\right)$ entre as diferentes tensōes consideradas: (a) entre $5 \times 10^{2}$ e $1 \times 10^{3}$; (b) entre $1 \times 10^{3}$ e $6 \times 10^{3}$; (c) entre $6 \times 10^{3}$ e $1 \times 10^{4}$; (d) entre $1 \times 10^{4}$ e $3 \times 10^{4} ;$ (e) entre $3 \times 10^{4}$ e $8 \times 10^{4}$; (f) entre $8 \times 10^{4}$ e $3 \times 10^{5}$ e (g) entre $3 \times 10^{5}$ e $1,5 \times 10^{6} \mathrm{~Pa}$. Tamanho da amostra: 250.

Sci. agric., Piracicaba, 50(3):383-392, out./dez., 1993

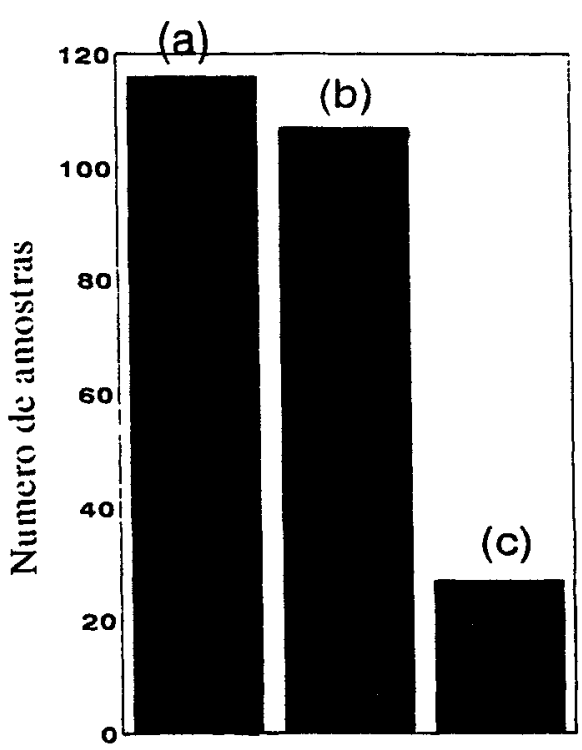

Figura 3. Comparação entre o número de amostras onde ocorreram valores de umidade correspondente a $3 \times 10^{5} \mathrm{~Pa}$ maiores (a), menores (b) $\mathrm{e}$ iguais (c) àquela correspondente a $1,5 \times 10^{6} \mathrm{~Pa}$.

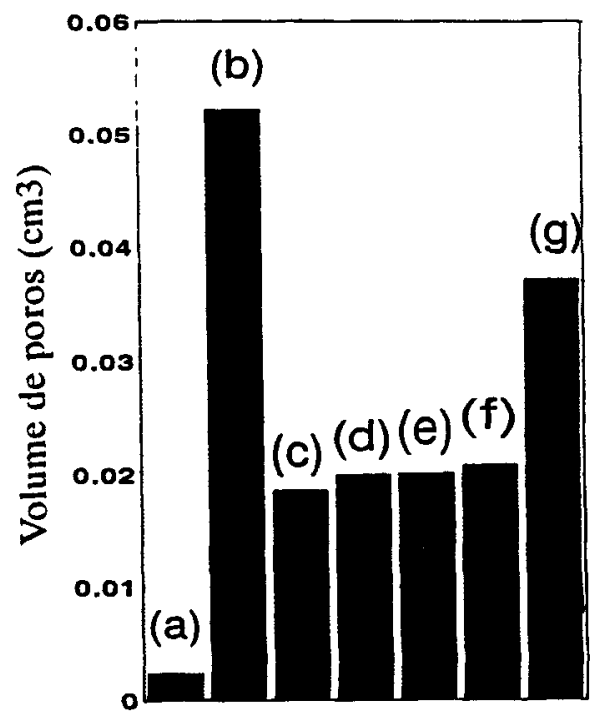

Figura 4. Volume de poros $\left(\mathrm{cm}^{3}\right)$ entre as diferentes tensões consideradas: (a) entre $5 \times 10^{2}$ e $1 \times 10^{3}$; (b) entre $1 \times 10^{3}$ e $6 \times 10^{3}$; (c) entre $6 \times 10^{3}$ e $1 \times 10^{4}$; (d) entre $1 \times 10^{4}$ e $3 \times 10^{4} ;(e)$ entre $3 \times 10^{4}$ e $8 \times 10^{4}$; (f) entre $8 \times 10^{4}$ e $3 \times 10^{5}$ e $(\mathrm{g})$ entre $3 \times 10^{5}$ e $1,5 \times 10^{6} \mathrm{~Pa}$. Tamanho da amostra: 143. 


$\begin{array}{lcccccccc}\text { Tensão: (PA) } & 5 \times 10^{2} & 1 \times 10^{3} & 6 \times 10^{3} & 1 \times 10^{4} & 3 \times 10^{4} & 8 \times 10^{4} & 3 \times 10^{5} & 1,5 \times 10^{6} \\ \text { Diâm: }(\mathrm{mm}) & 0,6 & 0,3 & 0,5 & 0,3 & 0,0098 & 0,0037 & 0,0010 & 0,002 \\ \text { Volume: }\left(\mathrm{cm}^{3}\right) & 0,002 & 0,053 & 0,016 & 0,022 & 0,026 & 0,039 & 0,014\end{array}$

Portanto uma terceira hipótese para a não retirada de água entre as tensões $5 \times 10^{2}$ e $1 \times 10^{3} \mathrm{~Pa} \epsilon$ apenas à inexistência ou pelo menos um diminuto volume de poros com diâmetro entre 0,6 e 0,3 $\mathrm{mm}$, no solo em questão.

Analisando-se um pouco mais as proporções entre os volumes descritos, distinguemse dois tipos de poros, um associado à retirada de água a baixas sucções, que seria devido a vazios interagregados e o segundo no qual a água estaria retida a maiores tensões, devido à vazios intraggregados (SHARMA \& UEHARA, 1968). A influência dos intraagregados na capacidade de retenção de água, poderia aumentar, aumentando-se a participação do volume de água retirada entre 3 $\times 10^{5} \mathrm{~Pa}$ e $1,5 \times 10^{6} \mathrm{~Pa}$, através de uma análise da Figura 3.

Isto é, os cálculos acima foram obtidos à partir dos valores médios das umidades, resumidos na TABELA 1, porém, considerando-se a Figura 3, que foi construida à partir dos dados originais (MORAES, 1991), pela observação e contagem do número de amostras onde ocorreram valores de umidade à base de volume correspondente a $3 \times 10^{5}$ $\mathrm{Pa}$ maiores, menores e iguais aos valores de umidade correspondente a $1,5 \times 10^{\circ} \mathrm{Pa}$ (respectivamente a, b e c na Figura 3) vê-se que para uma boa parte dos resultados, $\theta\left(3 \times 10^{5} \mathrm{~Pa}\right)$ $<\theta\left(1,5 \times 10^{6} \mathrm{~Pa}\right)$.

Se forem desprezados os valores de umidade correspondente a $3 \times 10^{5} \mathrm{~Pa}$ e $1,5 \times 10^{6} \mathrm{~Pa}$ destas 107 amostras, por representarem uma situação fisicamente impossível, isto é, uma pressão menor retirar mais água que a pressão maior, ter-se-ia a situação mostrada na Figura 4, que evidencia um aumento no volume de poros com água retida a tensões maiores que $3 \times 10^{5} \mathrm{~Pa}$, portanto uma maior influência dos vazios intraagregados na retenção de água.

Explorando um pouco mais estes resultados, com base na presença de distintas faixas de volume de poros intraagregados possivelmente atribuível ao grau de desenvolvimento estrutural ou anisotropia do solo, TSUJI et al. (1975), aliado ao fato de que Latossolos (Oxisol) desenvolvidos sob alta precipitação pluvial exibirão grande desenvolvimento estrutural (CAGAUAN \& UEHARA, 1965), calcularam-se as médias das umidades à base de volume às várias tensões para 116 amostras, isto $\hat{E}$, descartaram-se as amostras para as quais $\theta\left(3 \times 10^{5} \mathrm{~Pa}\right)=\theta\left(1,5 \times 10^{6} \mathrm{~Pa}\right)$ e $\theta$ $\left(3 \times 10^{5} \mathrm{~Pa}\right)<\theta\left(1,5 \times 10^{6} \mathrm{~Pa}\right)$, obtendo-se então os resultados mostrados na Figura 5.

Agora, o volume de poros responsável pela retenção de água com valores de potencial mátrico menores que $3 \times 10^{4} \mathrm{~Pa}$ é ainda maior, evidenciando ainda mais o caráter argiloso do solo em questão, fato este de fácil verificação não só pelo tipo de solo utilizado mas também pelos dados de PREVEDELLO (1987) que analisou 57 amostras de solo na mesma área do presente experimento.

É o momento de se indagar a qual ou quais fatores poder-se-ia atribuir os três tipos de resultados obtidos:

$\left.1^{\circ}\right) \theta\left(3 \times 10^{5} \mathrm{~Pa}\right)>\theta\left(1,5 \times 10^{6} \mathrm{~Pa}\right)$;

20) $\theta\left(3 \times 10^{5} \mathrm{~Pa}\right)=\theta\left(1,5 \times 10^{6} \mathrm{~Pa}\right)$;

$\left.3^{\circ}\right) \theta\left(3 \times 10^{5} \mathrm{~Pa}\right)<\theta\left(1,5 \times 10^{6} \mathrm{~Pa}\right)$.

O primeiro caso não constitui problema, aliás, seria o esperado. Para o $2^{\circ}$ e $3^{\circ}$ casos, as seguintes hipóteses são possiveis de terem ocorrido:

$\left.1^{2}\right)$ para o $2^{\circ}$ caso, $\theta\left(3 \times 10^{\prime} \mathrm{Pa}\right)=\theta(1,5 \times$ $10^{6} \mathrm{~Pa}$ ), exclusivamente, poder-se-ia lançar mão das considerações feitas no início da discussão, quando $\theta\left(5 \times 10^{2} \mathrm{~Pa}\right)=\theta\left(1 \times 10^{3} \mathrm{~Pa}\right)$ relativamente ao diâmetro de poros;

$\left.2^{2}\right)$ tempo de equilíbrio insuficiente (válida para o $2^{\circ}$ e $3^{\circ}$ casos);

3a) violação do equilíbrio termodinâmico (válida para $02^{\circ}$ e $3^{\circ}$ casos).

A primeira hipotese sugere que o menor diâmetro de poros acessível ao equipamento seria da ordem de $10 \times 10^{-4} \mathrm{~mm}$, correspondendo portanto a $3 \times 10^{5} \mathrm{~Pa}$; a aplicação desta pressão esgotaria o volume de água correspondente, da mesma maneira que a aplicação de $1,5 \times 10^{6} \mathrm{~Pa}$ isto 
$\varepsilon$, atuaria sobre o mesmo volume de poros, fazendo com que as umidades finais fossem iguais.

A expressão acessível ao equipamento refere-se ao fato de que poderiam haver poros menores, porém não compatíveis com a pressão máxima do equipamento que $\epsilon$ de $1,5 \times 10^{6} \mathrm{~Pa}$. Evidentemente esta hipótese não se aplica ao terceiro caso, pois uma maior pressão deveria ao menos explorar igual volume de poros como no segundo caso, mas nunca menor.

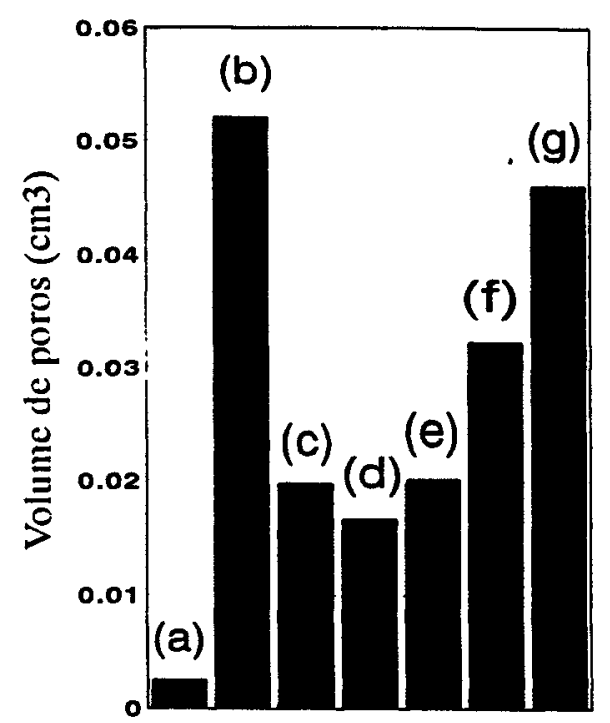

Figura 5 - Volume de poros $\left(\mathrm{cm}^{3}\right)$ entre as diferentes tensões consideradas: (a) entre $5 \times 10^{2}$ e $1 \times 10^{3}$; (b) entre $1 \times 10^{3}$ e $6 \times 10^{3}$; (c) entre $6 \times 10^{3}$ e $1 \times 10^{4}$; (d) entre $1 \times 10^{4} \mathrm{e}$ $3 \times 10^{4}$; (e) entre $3 \times 10^{4}$ e $8 \times 10^{4}$; (f) entre $8 \times 10^{4}$ e $3 \times 10^{5}$ e $(\mathrm{g})$ entre $3 \times 10^{5}$ e $1,5 \times 10^{6} \mathrm{~Pa}$. Tamanho da amostra: 116.

A segunda hipótese refere-se ao tempo que se aguarda para que o potencial mátrico da amostra se iguale ao de água livre, pela aplicação de uma pressão mais elevada Pk, GLOBUS et al. (1971).

Em média, o tempo para que as amostras entrassem em equilíbrio à pressão de $3 \mathrm{X}$ $10^{5} \mathrm{~Pa}$ foi de quinze dias, o que está de acordo com SOILMOISTURE EQUIPMENT CORP. (s.d.) que estima um tempo de cerca de dezoito dias em função da altura da amostra (cerca de $3 \mathrm{~cm}$ no caso). Já para 1,5 x $10^{6} \mathrm{~Pa}$ este tempo foi bastante variável, em função das amplitudes térmicas ocorridas no interior do laboratório. Assim, para pequenas amplitudes cerca de vinte dias foram suficientes, outras necessitaram quarenta $e$ cinco dias e em parte delas, o equilíbrio foi fixado em sete dias.

Então, a hipótese sugere que no primeiro caso o tempo esperado foi suficiente para que ocorresse o equilibrio, no segundo esteve próximo e no terceiro foi insuficiente. Convém ressaltar que embora fosse possível selecionar dois tempos (sete e vinte dias) para os três casos, praticamente $100 \%$ dos casos onde $\theta$ (3x $\left.10^{5} \mathrm{~Pa}\right)>\theta\left(1,5 \times 10^{6} \mathrm{~Pa}\right)$ ocorreu com tempos entre vinte $\mathrm{e}$ quarenta $\mathrm{e}$ cinco dias e quase $100 \%$ dos casos onde $\theta\left(3 \times 10^{5} \mathrm{~Pa} \leq \theta(1,5 \times\right.$ ${ }_{10}{ }^{5} \mathrm{~Pa}$ ) ocorreu para o tempo fixado de sete dias.

Esta ocorrencia maciça de valores $\theta$ (3 $\left.\times 10^{5} \mathrm{~Pa}\right) \leq \theta\left(1,5 \times 10^{6} \mathrm{~Pa}\right)$ para um tempo de equilibrio de sete dias embora reforce substancialmente a segunda hipbtese, não permite no entanto que se descarte a violação do equilíbrio termodinâmico, analisada a seguir.

Considere-se para tanto a equação:

$$
\Psi_{m}=v_{s} P_{s}+\int_{T_{3}}^{T_{2}}\left(s_{s}-s_{0}\right) d T
$$

onde:

$\boldsymbol{Y}_{\mathrm{m}}=$ potencial mátrico;

$\nabla_{s}=$ volume específico;

$\mathbf{P}_{\varepsilon}=$ pressão aplicada d̀ amostra de solo;

$s_{z}=$ entropia específica da solução no solo;

$s_{0}=$ entropia específica da solução à pressão atmosférica;

$T_{1}$ e $T_{2}=$ temperatura absoluta nos estados 1 e 2 respectivamente;

a qual, segundo GLOBUS et al. (1971), expressa o potencial mátrico de uma amostra não deformável, posicionada em uma câmara de pressão de Richards e hidraulicamente conectada e em equilibrio termodinâmico com a solução na câmara inferior cuja pressão relativa $\left(P_{\circ}\right)$ é constante $e$ igual à atmosférica, tomada como zero. 
Uma das condições de validade para o emprego da equação 1 , é que a pressão na câmara inferior permaneça constante enquanto o equilibrio é atingido.

No entanto, o longo tempo esperado para verificação visual do equilíbrio (vinte $e$ quarenta $e$ cinco dias para amostras que apresentaram $\theta\left(3 \times 10^{5} \mathrm{~Pa}\right)>\theta\left(1,5 \times 10^{6} \mathrm{~Pa}\right)$ chegando desaparecer a água do tubo de saída nas horas mais frias e depois aparecerem gotas nas horas mais quentes do dia indicariam que houve uma violação da condição de contorno inferior do sistema, isto é, a alta pressão, juntamente com as altas amplitudes térmicas facilitariam a difusão de gás para a parte inferior da placa, fazendo com que a base dos poros ao invés de estar em contato com água livre à pressão $P_{0}$, expulsasse a água sob a placa, levando então à formação de meniscos, diminuindo a área de fluxo, além de violar a condição de pressão $P_{0}$, GLOBUS et al. (1971). Esta lentidão no fluxo explicaria o aumento do tempo de vinte para quarenta e cinco dias, na época do ano de maior amplitude térmica (a variação de temperatura deveu-se à ausência de reguladores de temperatura do ar no laboratório, por ocasião do experimento, a questão da temperatura é particularmente crítica na região de Piracicaba, por ocasião do outono e inverno quando podem ocorrer grandes variações entre a máxima e a mínima em um mesmo dia).

- Ainda, se assumida a primeira hipótese, - efeito da temperatura dar-se-ia também na diminuição do teor de água a um dado potencial, devido à diminuição na tensão superficial, o que se daria a uma razão de cerca de $0,2 \%$ para cada variação de um grau na temperatura, DANIELSON \& SUTHERLAND (1986). Esta variação, explicaria também o fato de que muitas vezes se supunha o equilibrio mas depois surgiam gotas de água no vasilhame de coleta, nas horas mais quentes do dia. A aceitação da primeira hipótese levaria a que o menor teor de água nas amostras a $1,5 \times 10^{6} \mathrm{~Pa}$ deveu-se não à exploração do volume correspondente, mas a uma diminuição na energia de retenção devido à diferença de temperatura, ocasionando uma maior drenagem.

Embora a influência da temperatura sobre a tensão superficial possa ser significativa, por exemplo, à $10^{\circ} \mathrm{C}$ a tensão superficial água/ar é de $74,22 \times 10^{-3} \mathrm{~N} / \mathrm{m}$ caindo para $72,75 \times 10^{-3} \mathrm{~N} / \mathrm{m}$ a $25^{\circ} \mathrm{C}$, há os que contestam a intensidade desse efeito no sentido de explicar as diferenças observadas na relação $\Theta x \Psi m$ (PECK, 1960;
WILKINSON \& KLUTE, 1962; CHAHAL, 1963; CHAHAL, 1965 e CHAHAL, 1966), colocando como principal fator o efeito da temperatura sobre as bolhas de ar aprisionadas, principalmente nos solos de textura fina (WILKINSON \& KLUTE, 1962), como é o presente caso.

Infelizmente ocorreram bolhas de ar no presente trabalho mas felizmente puderam ser observadas para futuros cuidados e recomendações. Parte das bolhas são provenientes do próprio processo de saturação (WILKINSON \& KLUTE, 1962), que na verdade se tratou de uma embebição, atingindo-se então um valor de umidade $\theta_{0}<\theta_{\mathrm{S}}$ (KLUTE, 1986; BALL \& HUNTER, 1988), o que por si só significa presença de bolhas de ar. A observação das bolhas dava-se no momento da transferência das amostras para as placas definitivas isto é, as amostras eram saturadas em bandejas plásticas e depois levadas à câmara de pressão nas respectivas placas porosas, exceto no caso do funil de Haines. Quando as amostras eram retiradas das bandejas, o fundo delas, revestido de lenço de papel sofria um abaulamento devido ao peso da amostra. Imediatamente as amostras eram pressionadas sobre a placa a fim de que o contato fosse atingido. No ato de pressionar as amostras, evidentemente o abaulamento deixava de existir e a reação em sentido contrário, era de tal maneira que fazia com que algumas bolhas aprisionadas se manifestassem na superfície, tornando possível sua observação. A necessidade da saturação das amostras em bandejas e não nas próprias placas deveu-se ao seu número reduzido em função do número de amostras, problema esse a ser enfrentado quando o número de amostras é grande. De qualquer maneira a existência de bolhas é fato previsto quando se trabalha com embebição.

Outra consequência sensível das flutuações de temperatura é a destilação da água, tanto da placa porosa como das amostras. A água evapora e condensa-se nas paredes da câmara que contém o sistema placa-solo, levando a uma difícil identificação do equilibrio pela visualização do volume drenado, KLUTE (1986). Além disso, esta água evaporada talvez fosse significativa em termos do que se esperaria ser retirado pela aplicação de pressão, embora não se possa afirmar quanto, pois também água da placa se evapora. Isso reforça ainda mais a hipótese de violação do equilíbrio termodinâmico sob a placa, pela diminuição da água livre, agora não pela expulsão desta pelo gás mas sim por evaporação. 
KLUTE (1986) aconselha o uso de folhas de papel toalha umedecidas, não diretamente sobre as amostras, para que a evaporação se deva a essas folhas saturando a câmara e assim prevenindo a evaporação pela amostra. Deve-se ressaltar que este fenômeno de condensação nas paredes foi observado constantemente quando se trabalhou com tempos mais longos. Exemplificando, a pressão de saturação de vapor à $10^{\circ} \mathrm{C}$ é cerca de $9,21 \mathrm{~mm}$ de $\mathrm{Hg}$, atingindo 23,76 $\mathrm{mm}$ à $25^{\circ} \mathrm{C}$, fazendo com que se acentue a evaporação nas horas mais frias, quando se reduz a pressão de vapor.

As amplitudes térmicas ocorridas acarretam ainda um outro tipo de violação do equilíbrio termodinâmico, que pode ser verificado pela equação 1 . Uma vez que dT $\neq 0,0$ termo

$$
\int_{T_{1}}^{T_{2}}\left(S_{s}-S_{0}\right) d T
$$

teria que ser levado em conta no cálculo do $\psi \mathrm{m}$. Ainda, correções levando em conta as diferenças de temperatura ficam difíceis senão impossíveis, não só pelo desconhecimento de " $s$ " quanto à composição química mas também principalmente pela ausência de valores de entropia a baixas temperaturas $\mathrm{e}$ altas pressões $\left(1,5 \times 10^{6} \mathrm{~Pa}\right)$. Normalmente o que se tem devido à aplicações em engenharia é a entropia a altas pressões e acima de $100^{\circ} \mathrm{C}$, que não é o caso.

\section{CONCLUSÃO}

A variabilidade metodológica é bastante grande, principalmente a altas tensões e enquanto não se tem uma padronização universal ou, pelo menos nacional, deve-se optar pela curva de retenção completa (e não apenas a "capacidade de campo" e ao "ponto de murchamento permanente"), a fim de que se possa detectar estas incongruências, bem como o controle da temperatura na sala de operação do equipamento. São os cuidados mínimos que devem ser tomados a curto prazo.

\section{REFERENCIAS BIBLIOGRAFICAS}

BALL, B.C.; HUNTER, R. The determination of water release characteristics of soil cores at low suctions. Geoderma. Amsterdan, v.43, p.195-212, 1988.
BLAKE, G.R.; HARTGE, K.H. Bulk Density. In: BLACK, C.A., ed. Methods of Soil Analysis I. Physical and mineralogical methods. Madison: American Society of Agronomy, Soil Science Society of America, 1986. p. 363-375.

CAGAUAN, B.G.,; G. UEHARA. Soil anisotropy and its relation to aggregate stability. Soil Science Society of America Proceedings, Madison, v.29, p.198-200, 1965.

CHAHAL, R.S. Effect of temperature and trapped air on the energy status of water in porous media. Soil Science, Baltimore, v.98. p.107-112. 1963.

CHAHAL, R.S. Effect of temperature and trapped air on matric suction. Soil Science, Baltimore, v.100, p.262266. 1965.

CHAHAL, R.S. Effect of entrapped air and suction on matric suction. Soil Science, Baltimore. v.102, p.131134. 1966.

CHILDS, E.C. The use of soil moisture characteristics in soil studies. Soil Science, Baltimore. v.50, p.239-252. 1940.

DANIELSON, R.E.; SUTHERLAND, P.L. Porosity. In: BLACK, C.A., ed. Methods of Soil Analysis. I. Physical and mineralogical methods. Madison: American Society of Agronomy, Soil Science Society of America, 1986. p. 443-461.

EL-SWAIFY, S.A.; HENDERSON, D. W. Water retention by osmotic swelling of certain colloidal clays with varying ionic composition. Joumal of Soil Science, Oxford, v.18, p.223-232. 1967.

EMBRAPA, Serviço Nacional de Levantamento e Conservação de Solos. Manual de Métodos de Analise de Solo. Rio de Janeiro, 1979. 1v.

FREIRE, J.C. Retenção de umidade em perfil oxissol do município de Lavras, Minas Gerais. Piracicaba, 1975. 76 p. Dissertação (Mestrado) - Escola Superior de Agricultura "Luiz de Queiroz"/USP.

GLOBUS, A.M.; ROZENSHTOK, S.K.; SIROTKIN, V.M.; MICHURIN, B.N. Method of determining the soil moisture potencial with a pressure membrane apparatus. Soviet Soil Science. New York, v.3, p.90-98, 1971.

HAINES, W.B. Studies in the physical properties of soil. $V$. The hysteresis effect in capillary properties, and the modes of moisture distribution associated therewith. Joumal of Agricnltural Science. Cambridge, v.X, p.96-105, 1930.

KLUTE, A. Water retention: laboratory methods. In: BLACK, C.A., ed. Methods of Soil Analysis. I. Physical and mineralogical methods. Madison: American Society of Agronomy, Soil Science Society of America, 1986. p. 635-662. 
LEAMER, R.W.; LUTZ, J.F. Determination of poresize distribution in soils. Soll Science, Baltimore, v.49, p.347-361. 1940.

MORAES, S.O. Heterogeneidade hidráulica de uma terra roxa estruturada. Piracicaba, 1991. 141p. Tese (Doutorado) - Escola Superior de Agricultura "Luiz de Queiroz", Universidade de São Paulo.

MOTA, F.O.B. Retenção de água em perfil Alfissol do Municipio de Mossoró,RN. Piracicaba, 1976. 70 p. Dissertação (Mestrado) - Escola Superior de Agricultura "Luiz de Queiroz", Universidade de São Paulo.

PECK, A.J. Change of moisture tension with temperature and air pressure: thoretical. Soil Science, Baltimore, v.89, p.303-310. 1960.

PETERSEN, R.G.; CALVIN, L.D. Sampling. In: BLACK, C. A., ed. Methods of Soil Analysis. 1 . Physical and mineralogical methods. Madison: American Society of Agronomy, Soil Science Society of America, 1986. p.33-51.

PREVEDELLO, B. M.S. Variabilidade espacial de parâmetros de solo e planta. Piracicaba, 1987. 166 p. Tese (Doutorado) - Escola Superior de Agricultura "Luiz de Queiroz", Universidade de São Paulo.

REEVE, M.J.; SMITH, P.D.; THOMASSON, A.J. The effect of density on water retention properties of field soils. Journal of Soil Science, Oxford, v.24, p.355-367, 1973.

RICHARDS, L.A.; FIREMAN, M. Pressure-plate apparatus for measuring moisture sorption and transmission by soils. Soil Science, New Brunswick, v.56, p.395-404. 1943.

ROYER, J.M.; VACHAUD, G. Field determination of hysteresis in soil-water characteristics. Soil Science Society of America Proceedings. Madison, v.39, p.221-223, 1975.
SALTER, P.J.; WILLIAMS, J.B. The influence of texture on the moisture characteristics of soils. Part I: A critical comparison of techniques for determining the available water capacity and moisture characteristic curve of a soil. Journal of Soil Science, Oxford, 16, p.1-15, 1965 .

SHARMA, M.L.; UEHARA, G. Influence of soil structure on water relations in Low humic latosols. I. Water retention. Soil Science Society of America Proceedings, Madison, v.32. p.765-770. 1968.

SILVA, A.P. da. Variabilidade espacial de atributos físicos do solo. Piracicaba, 1988. 98 p. Tese (Doutorado) - Escola Superior de Agricultura "Luiz de Queiroz", Universidade de São Paulo.

SOILMOISTURE EQUIPMENT. Operating Instructions for the Cat. $n^{\circ} 1600,5$ bar pressure plate extraction. Santa Bárbara, s.d.

SOIL SURVEY STAFF, 1990. Keys to soil taxonomy, (SMSS Technical Monograph, 6). Blacksburg. 1990.

THOMAS, G.W.; MOODIE, J.E. Chemical relationships affecting the water-holding capacities of clays. Soil Science Society of America Proceedings, Madison v.33, p.645-651. 1962.

TSUJI, G.Y.; WATANABE, R.T.; SAKAI, S. Influence of soil microstructure on water characteristics of selected hawagiian soils. Soil Science Society America Proceedings, Madison, v.39, p.28-33, 1975.

WEBSTER, R. Quantitative and numerical methods in soil classification and survey. Oxford: Clarendon, 1977. 269p.

WILKINSON, G.E.; KLUTE, A. The temperature effect on the equilibrium energy status of water held by porous media. Soil Science Society Proceedings, Madison, v.26, p.326-329. 1962.

Enviado para publicação em 25.01.93

Aceito para publicação em 17.05.93 